\title{
Nonalcoholic Fatty Liver Disease and Urolithiasis. A Systematic Review and Meta-Analysis
}

\author{
Karn Wijarnpreecha ${ }^{1,2}$, Susan Lou ${ }^{3}$, Panadeekarn Panjawatanan ${ }^{4}$, Anawin Sanguankeo ${ }^{5,6}$, Surakit Pungpapong ${ }^{2}$, Frank J. \\ Lukens $^{2}$, Patompong Ungprasert ${ }^{7^{*}}$
}

1) Department of Internal

Medicine, Bassett Medical

Center, Cooperstown, NY, USA

2) Division of Gastroenterology

and Hepatology, Mayo Clinic

College of Medicine, Mayo

Clinic, Jacksonville, Florida,

USA

3) Department of Medicine,

University of Minnesota,

Minneapolis, MN, USA

4) Department of Biochemistry,

Faculty of Medicine, Chiang

Mai University, Chiang Mai,

Thailand

5) Divison of Nephrology,

Johns Hopkins University

School of Medicine, Baltimore,

MD, USA

6) Department of Preventive

and Social Medicine, Faculty

of Medicine, Siriraj Hospital,

Mahidol University, Bangkok,

Thailand

7) Clinical Epidemiology

Unit, Department of Research

and Development, Faculty

of Medicine Siriraj Hospital,

Mahidol University, Bangkok,

Thailand

\author{
Address for correspondence: \\ Patompong Ungprasert \\ 2 Wanglang Road, \\ Bangkoknoi, Bangkok, \\ Thailand 10700 \\ p.ungprasert@gmail.com
}

Received: 06.08.2018

Accepted: 03.12.2018

\section{ABSTRACT}

Background \& Aims: Recent studies have suggested that nonalcoholic fatty liver disease (NAFLD) could be a predisposing factor for urolithiasis but the results have been inconsistent. This systematic review and meta-analysis was conducted with the aim to summarize all available data.

Methods: A comprehensive literature review was conducted using MEDLINE and EMBASE databases through March 2018 to identify all studies that compared the risk of urolithiasis among patients with NAFLD versus those without NAFLD. Effect estimates from each study were extracted and combined together using the random-effect, generic inverse variance method of DerSimonian and Laird.

Results: A total of eight studies with 238,400 participants fulfilled the eligibility criteria and were included in the meta-analysis. The risk of urolithiasis among patients with NAFLD was significantly higher than in those without NAFLD with a pooled odds ratio of 1.81 (95\% confidence interval, 1.29-2.56; $\mathrm{I}^{2}$ 92\%).

Conclusions: A significantly increased risk of urolithiasis among patients with NAFLD was observed in this meta-analysis.

Key words: Nonalcoholic fatty liver disease - nonalcoholic steatohepatitis - urolithiasis - kidney stone meta-analysis.

Abbreviations: CT: computer tomography; NAFLD: nonalcoholic fatty liver disease.

\section{INTRODUCTION}

Nonalcoholic fatty liver disease (NAFLD) is a common disease characterized by fatty infiltration of the liver of greater than five percent of the liver weight that is not caused by other liver diseases or excessive alcohol consumption [1]. It has been estimated over 60 million people in the United States are affected by this disorder [2,3]. The pathogenesis of NAFLD is closely linked to chronic systemic inflammation and insulin resistance [4]. There are several known risk factors for NAFLD such as obesity, diabetes mellitus, hypertension, hyperuricemia, chronic kidney disease, physical inactivity and hypothyroidism [4-6].
Urolithiasis is one of the most common urological diseases caused by deposition of crystals in the renal medulla and genitourinary tract. Studies have demonstrated that the prevalence of urolithiasis in the United States increased from $4 \%$ in the year 1976 to 1980 to $8 \%$ in the year 2007 to $2010[7,8]$. Recent epidemiological studies have shown that urolithiasis is associated with several metabolic disorders, including obesity, diabetes mellitus and hypertension. Some authors even consider urolithiasis as a component of metabolic syndrome [9-12].

Recent studies have suggested that NAFLD could also be a risk factor for urolithiasis although the results varied considerably across the studies $[9,13-19]$. The current systematic review and meta-analysis was conducted with the aims to identify all studies that compared the risk of urolithiasis among patients with NAFLD versus individuals without NAFLD and to summarize the results of those studies together.

\section{METHODS}

Information Sources and Search strategy

A systematic literature search of the MEDLINE and EMBASE databases was carried out from inception to March 
2018 to identify all original studies that investigated the association between NAFLD and urolithiasis. The systematic literature review was independently conducted by three investigators (K.W., P.P., and P.U.) using a search strategy that included the terms for "nonalcoholic fatty liver disease", "steatohepatitis", "urolithiasis", "kidney stone", "ureteral stone", and "bladder stone" as described in online Supplementary Table I. No language limitation was applied. Abstracts of several prominent conferences, including Digestive Disease Week from 2010 to 2018, American College of Gastroenterology from 2004 to 2018, American Association for the Study of Liver Diseases from 2003 to 2018, United European Gastroenterology from 2009 to 2018 and European Congress of Radiology from 2003 to 2018 , were also searched. Hand-search was also performed on references of selected retrieved articles. The meta-analysis was conducted according to the PRISMA (Preferred Reporting Items for Systematic Reviews and Meta-Analysis) statement, which is provided as the online Supplementary Table II.

\section{Selection criteria}

To be eligible for the meta-analysis, studies had to be crosssectional, case-controlled or cohort studies that investigated the risk of urolithiasis among patients with NAFLD versus individuals without NAFLD. For the cohort study and crosssectional study, cases must be patients with NAFLD and comparators must be individuals without NAFLD. One of the reported outcomes must be urolithiasis (incident urolithiasis for cohort study and prevalent urolithiasis for cross-sectional study). For the case-control study, cases must be patients with urolithiasis and controls must be individuals without urolithiasis. The exposure of interest must be the history of NAFLD. Eligible studies must also provide the effect estimates [odds ratios (OR), relative risks (RR), hazard ratios (HR) or standardized incidence ratio (SIR)] with $95 \%$ confidence intervals (CI). Inclusion was not limited by study size. When more than one article utilizing the same database/cohort was available, only one study with the most comprehensive data/ analyses was included.

Retrieved articles were reviewed for their eligibility independently by the same three investigators (K.W., P.P., and P.U.) with disagreements resolved by consensus. The Newcastle-Ottawa quality assessment scale was used to appraise the quality of the studies in three domains, including the recruitment of participants, the comparability between the groups as well as the ascertainment of the outcome of interest for the cohort study and the ascertainment of the exposure of interest for the case-control study [20]. The modified Newcastle-Ottawa scale, described by Herzog et al., was used for the cross-sectional study [21].

\section{Data extraction}

The investigators used a structured information collection form to extract the following data from each study: title of the study, name of the first author, publication year, year of the study, country where the study was conducted, number of subjects, demographics of subjects, methods used to identify and verify NAFLD and urolithiasis, adjusted effect estimates with 95\% CI and covariates that were adjusted in the multivariable analysis.
To ensure accuracy, this data extraction process was independently performed by two investigators (K.W. and P.P.) and was reviewed by the senior investigator (P.U.).

\section{Statistical analysis}

Data analysis was performed using the Cochrane Collaboration's Review Manager 5.3 software (London, United Kingdom). Adjusted point estimates from each study were consolidated by the generic inverse variance method of DerSimonian and Laird, which assigned the weight of each study for the pooled analysis based on its variance [22]. As the outcome of interest was relatively uncommon, we planned to use the RR and HR of cohort studies as an estimate for the OR to calculate the pooled effect estimates with $\mathrm{OR}$ of case-control studies and cross-sectional studies. In light of the high probability of between-study variance because of different study designs, populations and methodologies, a random-effect model was used. Cochran's Q test and I2 statistic were used to quantify the between-study heterogeneity. A value of $\mathrm{I}^{2}$ of $0-25 \%$ represents insignificant heterogeneity, 26-50\% represents low heterogeneity, 51-75\% represents moderate heterogeneity, and more than $75 \%$ represents high heterogeneity [23]. Funnel plot was used for the assessment of the presence of publication bias.

\section{RESULTS}

A total of 347 potentially eligible articles were identified using the described search strategy (135 from Medline and 212 from EMBASE). After the exclusion of 128 duplicate articles, titles and abstracts of 219 unique articles were reviewed. One hundred and ninety-one articles were excluded at this stage since they were case reports, case series, correspondence, review articles, in vitro studies, animal studies or interventional studies, leaving 28 articles for full-text review: 18 of them were excluded after the full-length review, as they did not report the outcome of interest while 4 articles were excluded since they were descriptive studies without comparative analysis. Two conference abstracts were identified from searching of conference abstract databases. Finally 8 studies [9, 13-19] with 238,400 participants were included in the meta-analysis. The literature retrieval, review and selection process are shown in Fig. 1. The characteristics and quality appraisal of the included studies are presented in Table I. Inter-rater agreement for the quality assessment using the Newcastle-Ottawa scale was high with the kappa statistic of 0.80 .

\section{Association between nonalcoholic fatty liver disease and urolithiasis}

We found a significantly increased risk of urolithiasis among patients with NAFLD with the pooled OR of 1.81 (95\% CI), 1.29-2.56) as demonstrated in Fig. 2. The between-study heterogeneity was high with an $\mathrm{I}^{2}$ of $92 \%$.

\section{Subgroup analysis}

Subgroup analysis was conducted based on the methods used to diagnose urolithiasis (ultrasonography, computerized tomography $[\mathrm{CT}]$ and questionnaire) (Table I). The magnitude of the association was at the highest with the studies that used 


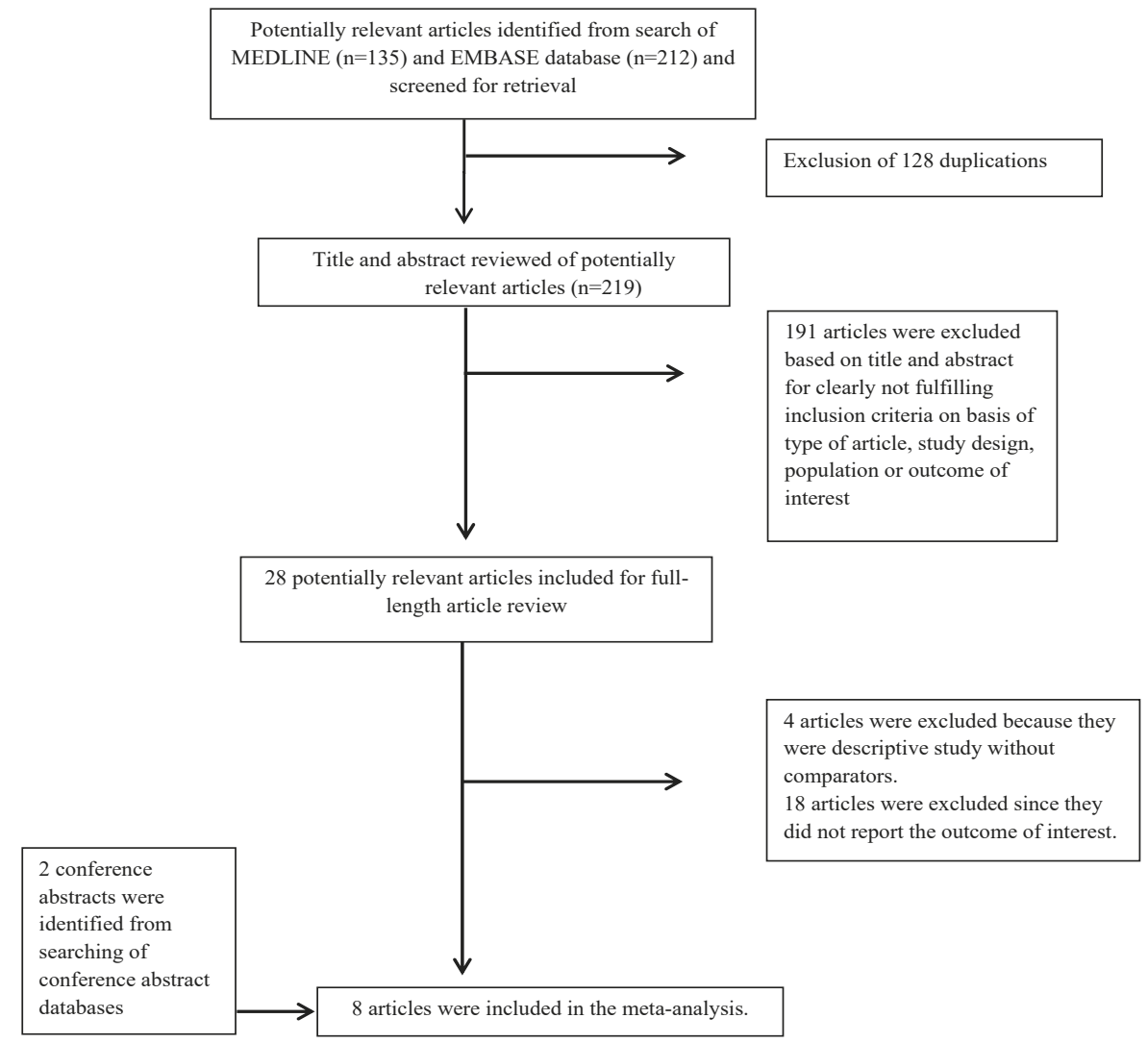

Fig. 1. Literature review process.

CT (pooled OR 2.83; 95\% CI, $1.14-7.00$; I2 90\%), followed by ultrasonography (pooled OR 1.49; 95\% CI, 0.79 - 2.78; I2 96\%) and a questionnaire (pooled OR 1.29; 95\% CI, $1.02-1.61$ ).

\section{Sensitivity analysis}

To further explore the high heterogeneity of this metaanalysis, a sensitivity analysis was performed by excluding the studies that did not adjust their effect estimates for comorbidities (i.e., excluding studies that did not adjust their results at all and studies that adjusted their results for only age and sex). A total of four studies [14, 17-19] remained in this sensitivity analysis and the new pooled OR decreased slightly to 1.21 (remained statistically significant with $95 \%$ CI of 1.00 - 1.45). Between-study statistical heterogeneity decreased to low level with $\mathrm{I}^{2}$ of $28 \%$.

\section{Evaluation for publication bias}

A funnel plot was constructed based on the effect estimate and accuracy of each study to assess for the presence of publication bias (Fig. 3). The plot is relatively asymmetric and is suggestive of the presence of publication bias in favor of positive studies.

\section{DISCUSSION}

The current systematic review and meta-analysis of studies on the association between NAFLD and risk of urolithiasis found an almost two-fold increased risk of urolithiasis among patients with NAFLD compared with individuals without NAFLD. The mechanism behind the increased risk is not known with certainty but there are a few possible explanations.
First, several classic metabolic risk factors for NAFLD, such as obesity, hypertension, diabetes mellitus and metabolic syndrome, have been increasingly recognized as predisposing factors for urolithiasis as well [24]. Studies have suggested that insulin resistance, the cornerstone of metabolic syndrome, may contribute to impaired ammonia synthesis by the kidneys. Insulin receptors are expressed in the renal tubular epithelium and insulin plays a significant role in the ammoniagenesis [25, 26]. Therefore, insulin resistance would lead to a decrease in urinary ammonia synthesis and excretion, resulting in lower urinary $\mathrm{pH}[10,25]$. In addition, patients with NAFLD are also often found to have hyperuricemia [6]. These two factors would serve as fertile ground for the formation of uric acid stones, although we do not have data from the primary studies whether the magnitude of the increased risk was more pronounced with uric acid stones. Obesity and insulin resistance may also directly alter the urine composition and increase the likelihood of stone formation. For instance, a large study of almost 6,000 participants found a higher concentration of metabolites and waste known to be associated with the formation of stones in the urine of obese participants [27].

Another possible explanation involves the role of inflammation and reactive oxygen species. Studies have suggested that membrane-bound vesicles from cells that die from oxidative stress could serve as the nidus for stone formation [28]. The kidney is an especially vulnerable organ to oxidative injury due to the abundance of long-chain polyunsaturated fatty acids in renal lipids [15]. Patients with NAFLD and metabolic syndrome are known to have a higher inflammatory burden and, therefore, a higher likelihood of renal oxidative cell death $[29,30]$. 


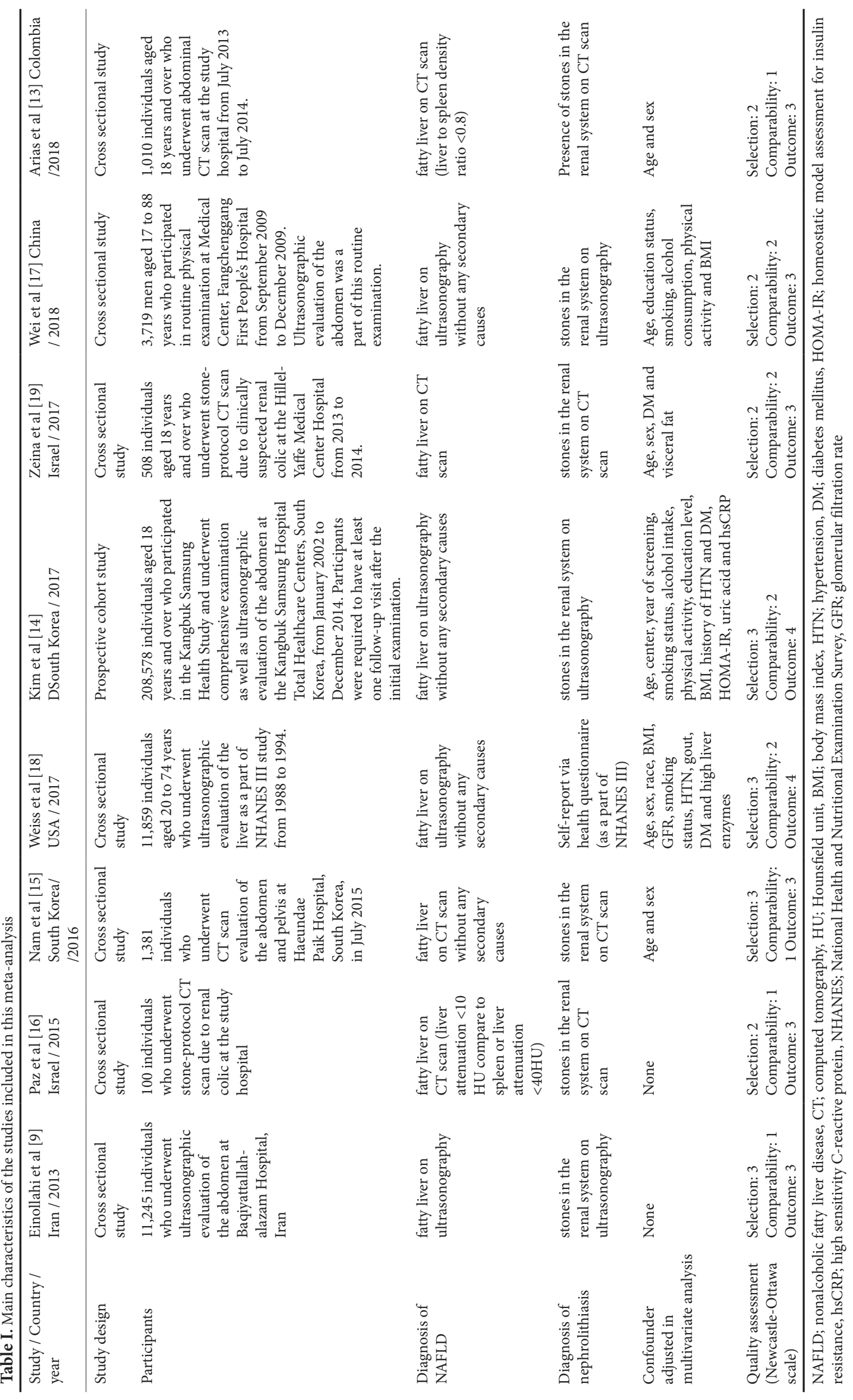




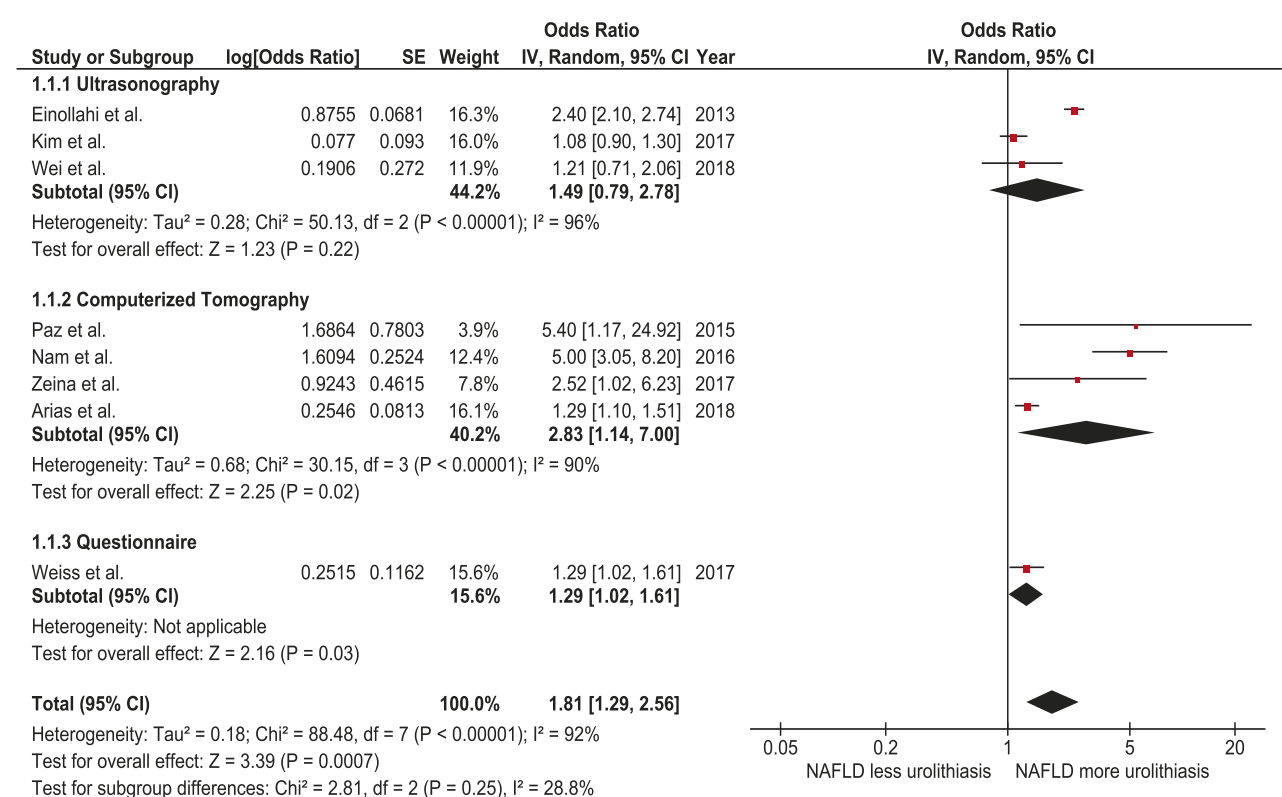

Fig. 2. Forest plot of all studies.

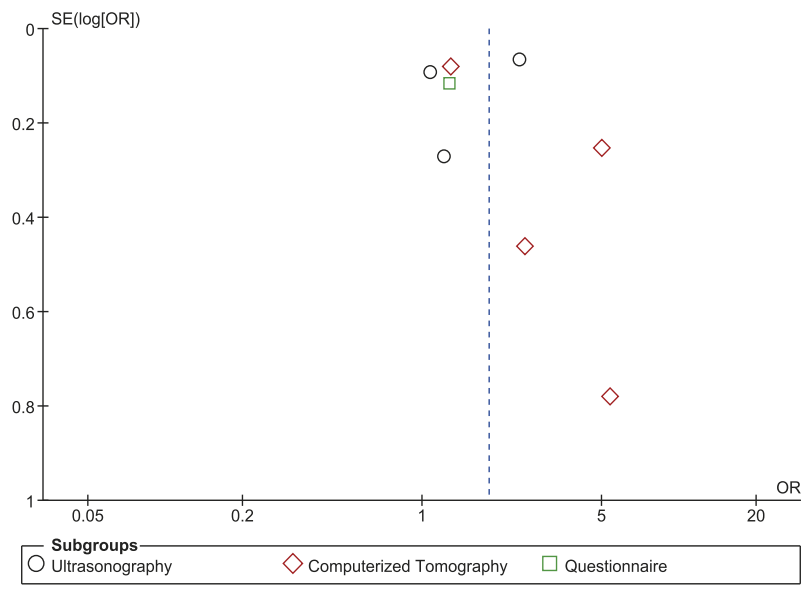

Fig. 3. Funnel plot of all studies.

Data on the association between the severity of NAFLD and the magnitude of the risk of urolithiasis are limited. Only one study investigated such a relationship. The authors divided NAFLD into mild, moderate and severe categories based on ultrasonographic appearance and found a significant correlation between the magnitude of the risk of urolithiasis and the severity of NAFLD ( $p$ for trend $=0.04$ ) [17].

Although the included studies were of high quality as reflected by their high Newcastle-Ottawa scores, we acknowledge that this systematic review and meta-analysis has some limitations and the results should be interpreted with caution.

First, statistical heterogeneity was high in the meta-analysis. We believe that the variation of quality of the effect estimates from each study was responsible for this as the sensitivity analysis that included only studies with more reliable results (i.e., studies that appropriately adjusted their effect estimates for potential confounders) showed $\mathrm{I}^{2}$ of only $28 \%$. There was also an inconsistency in the methods/criteria used for the diagnosis of NAFLD. Secondly, most of the included studies were cross- sectional studies. Therefore, the temporal relationship between NAFLD and urolithiasis could not be clearly established. Thirdly, the majority of the included studies were conducted in Asian countries, which may limit the generalizability of the observations to other populations. Fourthly, most of the primary studies included in this meta-analysis used ultrasonography to diagnose urolithiasis. Ultrasonography is a great tool to identify stones located in the kidney as well as pyeloureteral and vesicoureteric junctions but it often fails to detect ureteral calculi (unlike CT) [31]. Therefore, those studies may have missed some cases of urolithiasis, which may jeopardize the validity of their point estimates. This may also explain why subgroup analysis based on the methods used to diagnose urolithiasis found a higher magnitude of association among studies that diagnosed urolithiasis based on CT. Lastly, publication bias in favor of positive studies may have been present.

\section{CONCLUSION}

This study demonstrated a significantly increased risk of urolithiasis among patients with NAFLD. Insulin resistance, systemic inflammation and increased oxidative stress could be the link behind this association.

Conflicts of interest: We do not have any financial or non-financial potential conflicts of interest.

Authors' contributions. K.W., P.U. conceived and designed the study. K.W., S.L., P.P. and S.P. collected the data, K.W., P.P. and A.S. analyzed and interpreted the data. K.W., S.L. drafted the article. A.S., S.P., F.L. revised the article. K.W., S.L., P.P., A.S., S.P., F.L.and P.U. approved the final version of the manuscript. P.U. revised critically the manuscript.

Supplementary material: To access the supplementary material visit the online version of the J Gastrointestin Liver Dis at http://www. 
jgld.ro/wp/archive/y2018/n4/a13 and http://dx.doi.org/10.15403/ jgld.2014.1121.274.nac

\section{REFERENCES}

1. Review T, LaBrecque DR, Abbas Z, et al. World Gastroenterology Organisation global guidelines: Nonalcoholic fatty liver disease and nonalcoholic steatohepatitis. J Clin Gastroenterol 2014;48:467-473. doi:10.1097/MCG.0000000000000116

2. Sayiner M, Koenig A, Henry L, Younossi ZM. Epidemiology of Nonalcoholic Fatty Liver Disease and Nonalcoholic Steatohepatitis in the United States and the Rest of the World. Clin Liver Dis 2016;20:205214. doi:10.1016/j.cld.2015.10.001

3. Younossi ZM, Blissett D, Blissett R, et al. The economic and clinica burden of nonalcoholic fatty liver disease in the United States and Europe. Hepatology. 2016;64:1577-1586. doi:10.1002/hep.28785

4. Byrne CD, Targher G. NAFLD: a multisystem disease. J Hepatol 2015;62(1 Suppl):S47-S64. doi:10.1016/j.jhep.2014.12.012

5. Al-Dayyat HM, Rayyan YM, Tayyem RF. Non-alcoholic fatty liver disease and associated dietary and lifestyle risk factors. Diabetes Metab Syndr 2018;12:569-575. doi:10.1016/j.dsx.2018.03.016

6. Wijarnpreecha K, Panjawatanan P, Lekuthai N, Thongprayoon C, Cheungpasitporn W, Ungprasert P. Hyperuricaemia and risk of nonalcoholic fatty liver disease: A meta-analysis. Liver Int 2017;37:906 918. doi:10.1111/liv.13329

7. Scales CD Jr, Smith AC, Hanley JM, Saigal CS; Urologic Diseases in America Project. Prevalence of kidney stones in the United States. Eur Urol 2012;62:160-165. doi:10.1016/j.eururo.2012.03.052

8. Stamatelou KK, Francis ME, Jones CA, Nyberg LM, Curhan GC. Time trends in reported prevalence of kidney stones in the United States: 1976-1994. Kidney Int 2003;63:1817-1823. doi:10.1046/j.15231755.2003.00917.x

9. Einollahi B, Naghii MR, Sepandi M. Association of nonalcoholic fatty liver disease (NAFLD) with urolithiasis. Endocr Regul 2013;47:27-32. doi:10.4149/endo_2013_01_27

10. Spatola L, Ferraro PM, Gambaro G, Badalamenti S, Dauriz M. Metabolic Syndrome and Uric Acid Nephrolithiasis: Insulin Resistance in Focus. Metabolism 2018;83:225-233. doi:10.1016/j.metabol.2018.02.008

11. Taylor EN, Stampfer MJ, Curhan GC. Obesity, weight gain, and the risk of kidney stones. JAMA 2005;293:455-462. doi:10.1001/jama.293.4.455

12. Taylor EN, Stampfer MJ, Curhan GC. Diabetes mellitus and the risk of nephrolithiasis. Kidney Int 2005;68:1230-1235. doi:10.1111/j.15231755.2005.00516.x

13. Arias CLF, Ramirez L, Ortiz O. Renal lithiasis and Fatty Liver Disease, is there any relationship? European Congress of Radiology (ECR). 2018; Poster No C-3221.

14. Kim S, Chang Y, Sung E, et al. Non-alcoholic fatty liver disease and the development of nephrolithiasis: A cohort study. PloS One 2017;12:e0184506. doi:10.1371/journal.pone.0184506

15. Nam IC. Association of non-alcoholic fatty liver disease with renal stone disease detected on computed tomography. Eur J Radiol Open 2016;3:195-199. doi:10.1016/j.ejro.2016.07.004
16. Paz D GL. Association of renal stone (urolithiasis) with nonalcoholic fatty liver (NAFL). European Congress of Radiology (ECR). 2015; Poster No C-2056.

17. Wei YP, Lin XG, He RQ, et al. Epidemiologic Association of Nonalcoholic Fatty Liver Disease and Urinary Calculi: a Populationbased Cross-sectional Study in Southern China. Iran J Kidney Dis 2018;12:112-119.

18. Weiss A, Gaughan J, Ghiraldi E, Deitch C, Friedlander J. Independent association between nonalcoholic fatty liver disease and nephrolithiasis. Gastroenterology 2017;152 Supplement 1:S688.

19. Zeina AR, Goldenberg L, Nachtigal A, Hasadia R, Saliba W. Association between nephrolithiasis and fatty liver detected on non-enhanced CT for clinically suspected renal colic. Clin Imaging 2017;43:148-152. doi:10.1016/j.clinimag.2017.03.011

20. Wells GA, Shea B, O'Connell D, et al. The Newcastle-Ottawa Scale (NOS) for assessing the quality of nonrandomised studies in meta-analyses. Available at: http://www.ohri.ca/programs/clinical_epidemiology/ oxford.asp

21. Herzog R, Alvarez-Pasquin MJ, Diaz C, Del Barrio JL, Estrada JM, Gil A. Are healthcare workers' intentions to vaccinate related to their knowledge, beliefs and attitudes? A systematic review. BMC Public Health 2013;13:154. doi:10.1186/1471-2458-13-154

22. DerSimonian R, Laird N. Meta-analysis in clinical trials. Control Clin Trials 1986;7:177-188. doi:10.1016/0197-2456(86)90046-2

23. Higgins JP, Thompson SG, Deeks JJ, Altman DG. Measuring inconsistency in meta-analyses. BMJ 2003;327:557-560. doi:10.1136/ bmj.327.7414.557

24. Besiroglu H, Otunctemur A, Ozbek E. The metabolic syndrome and urolithiasis: a systematic review and meta-analysis. Ren Fail 2015;37:1-6. doi:10.3109/0886022X.2014.976133

25. Abate N, Chandalia M, Cabo-Chan AV Jr, Moe OW, Sakhaee K. The metabolic syndrome and uric acid nephrolithiasis: novel features of renal manifestation of insulin resistance. Kidney Int 2004;65:386-392. doi:10.1111/j.1523-1755.2004.00386.x

26. Klisic J, Hu MC, Nief $\mathrm{V}$, et al. Insulin activates $\mathrm{Na}(+) / \mathrm{H}(+)$ exchanger 3 : biphasic response and glucocorticoid dependence. Am J Physiol Renal Physiol 2002;283:F532-F539. doi:10.1152/ajprenal.00365.2001

27. Powell CR, Stoller ML, Schwartz BF, et al. Impact of body weight on urinary electrolytes in urinary stone formers. Urology 2000;55:825-830. doi:10.1016/S0090-4295(99)00617-2

28. Khan SR, Glenton PA, Backov R, Talham DR. Presence of lipids in urine, crystals and stones: implications for the formation of kidney stones. Kidney Int 2002;62:2062-2072. doi:10.1046/j.15231755.2002.00676.x

29. Arrese M, Cabrera D, Kalergis AM, Feldstein AE. Innate Immunity and Inflammation in NAFLD/NASH. Dig Dis Sci 2016;61:1294-1303. doi:10.1007/s10620-016-4049-X

30. Ganz M, Szabo G. Immune and inflammatory pathways in NASH Hepatol Int 2013;7 Suppl 2:771-781. doi:10.1007/s12072-013-9468-6

31. Turk C, Petrik A, Sarica K, et al. EAU Guidelines on Diagnosis and Conservative Management of Urolithiasis. Eur Urol 2016;69:468-474. doi:10.1016/j.eururo.2015.07.040 


\section{Online Supplementary Data}

\section{Search Strategy}

Database: Ovid MEDLINE

1. Nonalcoholic fatty liver.mp. or exp Nonalcoholic fatty liver disease/

2. fatty liver.mp. or exp fatty liver/

3. nonalcoholic steatohepatitis.mp.

4. steatohepatitis.mp.

5. or/ $1-4$

6. urolithiasis.mp. or exp urolithiasis/

7. urinary calculi.mp. or exp urinary calculi/

8. kidney calculi.mp. or exp kidney calculi/

9. nephrolithiasis.mp. or exp nephrolithiasis/

10. bladder stone.mp. or exp urinary bladder calculi/

11. staghorn stone.mp.

12. ureteral calculi.mp. or exp ureteral calculi/

13. calcium oxalate stone.mp. or exp calcium oxalate/

14. or/6-13

15.5 and 14 
Database: EMBASE

1. Nonalcoholic fatty liver or Nonalcoholic fatty liver/exp

2. fatty liver.mp. or exp fatty liver/

3. nonalcoholic.mp. AND (steatohepatitis.mp. or exp steatohepatitis/)

4. steatohepatitis.mp. or exp steatohepatitis/

5. or/1-4

6. urolithiasis.mp. or exp urolithiasis/

7. nephrolithiasis.mp. or exp nephrolithiasis/

8. bladder stone.mp. or exp bladder stone/

9. staghorn stone.mp. or exp staghorn stone/

10. ureter stone.mp. or exp ureter stone/

11. calcium oxalate stone.mp. or exp calcium oxalate stone/

12. or/6-11

13. 5 and 12 
PRISMA 2009 Checklist

\begin{tabular}{|c|c|c|c|}
\hline Section/topic & $\#$ & Checklist item & $\begin{array}{l}\text { Reported } \\
\text { on page }\end{array}$ \\
\hline \multicolumn{4}{|l|}{ TITLE } \\
\hline Title & 1 & Identify the report as a systematic review, meta-analysis, or both. & 1 \\
\hline \multicolumn{4}{|l|}{ ABSTRACT } \\
\hline Structured summary & 2 & $\begin{array}{l}\text { Provide a structured summary including, as applicable: background; objectives; data sources; study eligibility criteria, } \\
\text { participants, and interventions; study appraisal and synthesis methods; results; limitations; conclusions and } \\
\text { implications of key findings; systematic review registration number. }\end{array}$ & 2 \\
\hline \multicolumn{4}{|l|}{ INTRODUCTION } \\
\hline Rationale & 3 & Describe the rationale for the review in the context of what is already known. & 3 \\
\hline Objectives & 4 & $\begin{array}{l}\text { Provide an explicit statement of questions being addressed with reference to participants, interventions, comparisons, } \\
\text { outcomes, and study design (PICOS). }\end{array}$ & 3 \\
\hline \multicolumn{4}{|l|}{ METHODS } \\
\hline Protocol and registration & 5 & $\begin{array}{l}\text { Indicate if a review protocol exists, if and where it can be accessed (e.g., Web address), and, if available, provide } \\
\text { registration information including registration number. }\end{array}$ & 4 \\
\hline Eligibility criteria & 6 & $\begin{array}{l}\text { Specify study characteristics (e.g., PICOS, length of follow-up) and report characteristics (e.g., years considered, } \\
\text { language, publication status) used as criteria for eligibility, giving rationale. }\end{array}$ & 4 \\
\hline Information sources & 7 & $\begin{array}{l}\text { Describe all information sources (e.g., databases with dates of coverage, contact with study authors to identify } \\
\text { additional studies) in the search and date last searched. }\end{array}$ & 4 \\
\hline Search & 8 & $\begin{array}{l}\text { Present full electronic search strategy for at least one database, including any limits used, such that it could be } \\
\text { repeated. }\end{array}$ & 4 \\
\hline Study selection & 9 & $\begin{array}{l}\text { State the process for selecting studies (i.e., screening, eligibility, included in systematic review, and, if applicable, } \\
\text { included in the meta-analysis). }\end{array}$ & $4-5$ \\
\hline Data collection process & 10 & $\begin{array}{l}\text { Describe method of data extraction from reports (e.g., piloted forms, independently, in duplicate) and any processes } \\
\text { for obtaining and confirming data from investigators. }\end{array}$ & $4-5$ \\
\hline Data items & 11 & $\begin{array}{l}\text { List and define all variables for which data were sought (e.g., PICOS, funding sources) and any assumptions and } \\
\text { simplifications made. }\end{array}$ & $4-5$ \\
\hline Risk of bias in individual studies & 12 & $\begin{array}{l}\text { Describe methods used for assessing risk of bias of individual studies (including specification of whether this was } \\
\text { done at the study or outcome level), and how this information is to be used in any data synthesis. }\end{array}$ & Table 1 \\
\hline Summary measures & 13 & State the principal summary measures (e.g., risk ratio, difference in means). & 5 \\
\hline Synthesis of results & 14 & $\begin{array}{l}\text { Describe the methods of handling data and combining results of studies, if done, including measures of consistency } \\
\left(\text { e.g., }\left.\right|^{2}\right) \text { for each meta-analysis. }\end{array}$ & $5-6$ \\
\hline
\end{tabular}




\section{gasting PRISMA 2009 Checklist}

\begin{tabular}{|c|c|c|c|}
\hline Section/topic & $\#$ & Checklist item & $\begin{array}{l}\text { Reported } \\
\text { on page \# }\end{array}$ \\
\hline Risk of bias across studies & 15 & $\begin{array}{l}\text { Specify any assessment of risk of bias that may affect the cumulative evidence (e.g., publication bias, selective } \\
\text { reporting within studies). }\end{array}$ & $5-6$ \\
\hline Additional analyses & 16 & $\begin{array}{l}\text { Describe methods of additional analyses (e.g., sensitivity or subgroup analyses, meta-regression), if done, indicating } \\
\text { which were pre-specified. }\end{array}$ & $5-6$ \\
\hline \multicolumn{4}{|l|}{ RESULTS } \\
\hline Study selection & 17 & $\begin{array}{l}\text { Give numbers of studies screened, assessed for eligibility, and included in the review, with reasons for exclusions at } \\
\text { each stage, ideally with a flow diagram. }\end{array}$ & 6 \\
\hline Study characteristics & 18 & $\begin{array}{l}\text { For each study, present characteristics for which data were extracted (e.g., study size, PICOS, follow-up period) and } \\
\text { provide the citations. }\end{array}$ & Table 1 \\
\hline Risk of bias within studies & 19 & Present data on risk of bias of each study and, if available, any outcome level assessment (see item 12). & Table 1 \\
\hline Results of individual studies & 20 & $\begin{array}{l}\text { For all outcomes considered (benefits or harms), present, for each study: (a) simple summary data for each } \\
\text { intervention group (b) effect estimates and confidence intervals, ideally with a forest plot. }\end{array}$ & Figure 2 \\
\hline Synthesis of results & 21 & Present results of each meta-analysis done, including confidence intervals and measures of consistency. & $6-7$ \\
\hline Risk of bias across studies & 22 & Present results of any assessment of risk of bias across studies (see Item 15). & 7 \\
\hline Additional analysis & 23 & Give results of additional analyses, if done (e.g., sensitivity or subgroup analyses, meta-regression [see Item 16]). & $\begin{array}{l}\text { Not } \\
\text { applicable }\end{array}$ \\
\hline \multicolumn{4}{|l|}{ DISCUSSION } \\
\hline Summary of evidence & 24 & $\begin{array}{l}\text { Summarize the main findings including the strength of evidence for each main outcome; consider their relevance to } \\
\text { key groups (e.g., healthcare providers, users, and policy makers). }\end{array}$ & $7-8$ \\
\hline Limitations & 25 & $\begin{array}{l}\text { Discuss limitations at study and outcome level (e.g., risk of bias), and at review-level (e.g., incomplete retrieval of } \\
\text { identified research, reporting bias). }\end{array}$ & 8 \\
\hline Conclusions & 26 & Provide a general interpretation of the results in the context of other evidence, and implications for future research. & 8 \\
\hline \multicolumn{4}{|l|}{ FUNDING } \\
\hline Funding & 27 & $\begin{array}{l}\text { Describe sources of funding for the systematic review and other support (e.g., supply of data); role of funders for the } \\
\text { systematic review. }\end{array}$ & 9 \\
\hline
\end{tabular}

